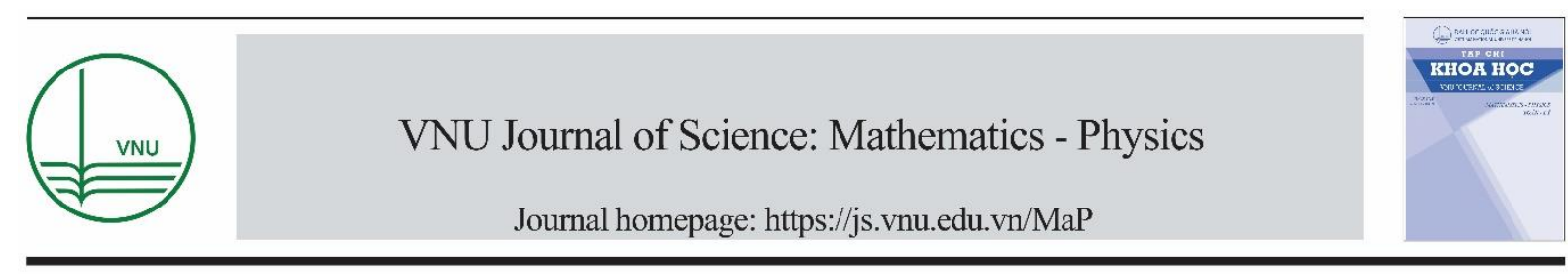

Original Article

\title{
High-pressure Effect of Elastic and Mechanical Properties of Hexagonal Gallium Nitride
}

\author{
Nenuwe* , O.N, Agbalagba O.E \\ Federal University of Petroleum Resources, P. M. B. 1221, Effurun, Delta State, Nigeria
}

Received 11 June 2020

Revised 30 June 2020; Accepted 15 July 2020

\begin{abstract}
This study examines an effect of pressure up to $50 \mathrm{GPa}$ on the elastic and mechanical properties of wurtzite gallium nitride ( $\mathrm{w}-\mathrm{GaN}$ ) by using classical potential within the Atomistic Tool Kit (ATK)-force field. The obtained results show that the elastic constants and other related parameters, such as Young's modulus, shear modulus, bulk modulus, Poisson's ratio, Pugh's ratio, Zener anisotropy factor and Kleinman parameter increase monotonically with increase of pressure up to $32 \mathrm{GPa}$. Beyond this pressure, we observed a non-linear behavior with increase in pressure. This might be attributed to the phase transition in GaN in the pressure range of 33.4 - $44.6 \mathrm{GPa}$. The results obtained for zero pressure are consistent with both experimental data and the theoretical data shown in references.
\end{abstract}

Keywords: Elastic constants, mechanical properties, wurtzite gallium nitride, high-pressure.

\section{Introduction}

Gallium nitride is a promising III-V semiconductor material, which has been found to be very useful in the manufacture of high power and high frequency optoelectronic devices [1]. It exhibits very remarkable physical properties, such as high thermal conductivity, low dielectric constants, high melting point, large bulk modulus, wide band gap, etc. [2-4]. This has attracted the attention of both theoretical and experimental researchers, into the study of $\mathrm{GaN}$ material $[1,5,6]$. Another vital attribute of gallium nitride is that, under ambient conditions, GaN is crystallized in hexagonal wurtzite phase. However, at high pressure the $\mathrm{w}-\mathrm{GaN}$ is known to transform to rock-salt phase, thereby exhibiting different mechanical properties [4].

\footnotetext{
* Corresponding author.

Email address: nenuwe.nelson@fupre.edu.ng
}

https//doi.org/ 10.25073/2588-1124/vnumap.4555 
Over the years, scientists have studied several exciting properties of gallium nitride [7-10]. In particular, Saoud et al. [4] reported wurtzite phase transition to rock-salt phase and from rock-salt phase to zincblende phase of $\mathrm{GaN}$ ( $\mathrm{z}-\mathrm{GaN}$ ) under a pressure up to 100GPa. Phase transition of GaN under pressure was studied by Li-Na et al. [11], using first principle DFT calculations. The obtained results were consistent with both experimental and theoretical data. In [8], Wright reported the elastic properties of z-GaN and hexagonal AlN, GaN and InN. By using DFT and plane-wave calculations, the author [8] obtained elastic properties of these materials at zero pressure. Sherwin and Drummond [12] calculated the stiffness constants of zincblende group III-nirides from the stiffness constants of the wurtzite phase, and obtained fairly good results. Polian et al. [10] studied elastic constants of $\mathrm{GaN}$ at zero pressure, using the Brillouin scattering experimental method. They obtained results differ substantially from data in the literature. Yamaguchi et al. [5] measured the stiffness constants of $\mathrm{GaN}$ at zero pressure, the obtained values were consistent with theoretical ones.

Although, there have been few studies of elastic properties of $\mathrm{GaN}$ at zero and applied pressures, experimental and theoretical studies of elastic and mechanical properties of $\mathrm{w}-\mathrm{GaN}$ under high-pressure are very limited. Therefore, the main goal of this work is to give a comprehensive study of the elastic and mechanical properties of $\mathrm{GaN}$ in wurtzite phase under high-pressure. In this paper, the elastic constants, Young's modulus, shear modulus, bulk modulus, Poisson's ratio, Pugh's ratio, Zener anisotropy factor and Kleinman parameter are calculated and analyzed in detail for this material up to $50 \mathrm{GPa}$. These elastic parameters of GaN are investigated by the ATK-force field method.

\section{Material and Methods}

The computation of elastic parameters of GaN was performed using ATK-force field code with Tersoff potentials [13] as implemented in QuantumATK under the framework of Virtual Nano Lab [14]. In this method, QuantumATK employs the Lagrangian strain and stress tensors. First, the atomic positions of each strained cell are optimized. Then, QuantumATK uses the universal linearlyindependent coupling strain vectors to minimize the number of stress calculations. For each strain vector, three deformations $(-\eta, 0,+\eta)$ centered at $\eta=0$, are applied to simulate the cell along selected strains and calculate the corresponding stress vectors. Here, we use $\eta=0.002$, and number of intermediate deformations $n_{\eta}=3$ to filter out possible non-linear contributions. The highest polynomial order was taken as one in the stress against $\eta$ fitting. Contributions from the linear stress are obtained by fitting the stress $\sigma_{i}(\eta)$ curves of each Voigt stress and for every strain component. Then, taking crystal symmetry into account, the independent stiffness constants are calculated as the least-squares solution to a linear system of equations. The calculations were performed within a range of pressures, 0 $-50 \mathrm{GPa}$.

\section{Results and Discussion}

In this section, we present the calculation of the elastic constants and other related elastic parameters of w-GaN at zero and applied pressures. In Table1, we summarized our results and experimental values as well as other theoretical results obtained at zero pressure. Results obtained for $C_{11}, C_{33}, C_{44}$ and $C_{66}$ are relatively lower than both experimental and theoretical values except for the data obtained by Savastenko and Sheleg [15] for $C_{11}$. The deviation between our results and the Brillouin scattering experimental data [5] are $5.1 \%, 0.16 \%, 22.5 \%$, and $17.9 \%$, respectively. While, $C_{12}$ is higher than 
experimental value by $13.2 \%$ and $C_{13}$ is in perfect agreement with experimental value. The calculated bulk modulus at zero pressure is in agreement with both experiment and theoretical data. The deviations observed for $C_{i j}$ are basically due to the potential force field used in our calculation. However, bearing in mind the theoretical approximations and experimental uncertainties, one can say that our calculated values for $C_{i j}$ are consistent with the experiment and theoretical values reported in literature.

According to the symmetry of crystals, hexagonal structures have five independent elastic constants $C_{\mathrm{ij}}\left(C_{11}, C_{12}, C_{13}, C_{33}, C_{44}\right)$. The mechanical stability for hexagonal crystal is determined by the following conditions $[16,17]$ :

$$
\begin{aligned}
& C_{11}>\left|C_{12}\right| ; \quad C_{44}>0 ; \quad C_{33}\left(C_{11}+C_{12}\right)>2 C_{13}^{2} ; C_{66}>0, \\
& \text { where, } C_{66}=\frac{1}{2}\left(C_{11}-C_{12}\right)
\end{aligned}
$$

Elastic constants obtained in this work for w-GaN at zero pressure satisfy the mechanical stability conditions given by Eq. (1). Our results indicate that $\mathrm{w}-\mathrm{GaN}$ is mechanically stable at zero pressure.

Table 1. Calculated elastic constants $\mathrm{C}_{\mathrm{ij}}$ (in GPa), Young's modulus E (in GPa), shear modulus G (in GPa), bulk modulus B (in GPa), Pugh's ratio (v), Zener anisotropy factor (A) and Kleinman parameter $(\zeta$ )

for $\mathrm{w}-\mathrm{GaN}$ at zero pressure.

\begin{tabular}{|l|l|l|l|}
\hline & This work & Other calculations & Experiment \\
\hline $\boldsymbol{C}_{\mathbf{1 1}}$ & 344.46 & $356^{\mathrm{a}}, 367^{\mathrm{b}}, 396^{\mathrm{c}}, 26^{\mathrm{d}}$ & $375-405^{\mathrm{e}}, 363-367^{\mathrm{m}}$ \\
\hline $\boldsymbol{C}_{\mathbf{1 2}}$ & 157.43 & $146^{\mathrm{a}}, 135^{\mathrm{b}}, 144^{\mathrm{c}}, 130^{\mathrm{d}}$ & $125-165^{\mathrm{e}}, 131-139^{\mathrm{m}}$ \\
\hline $\boldsymbol{C}_{\mathbf{1 3}}$ & 122.69 & $115^{\mathrm{a}}$ & $86-126^{\mathrm{e}}, 98-130^{\mathrm{m}}$ \\
\hline $\boldsymbol{C}_{\mathbf{3 3}}$ & 379.36 & $382^{\mathrm{a}}$ & $378-418^{\mathrm{e}}, 380-382^{\mathrm{m}}$ \\
\hline $\boldsymbol{C}_{\mathbf{4 4}}$ & 82.15 & $91^{\mathrm{a}}$ & $95-115^{\mathrm{e}}, 106-112^{\mathrm{m}}$ \\
\hline $\boldsymbol{C}_{\mathbf{6 6}}$ & 93.52 & $105^{\mathrm{a}}$ & $113-133^{\mathrm{e}}, 114-116^{\mathrm{m}}$ \\
\hline $\mathbf{B}$ & 208.20 & $215.41^{\mathrm{f}}, 196.21^{\mathrm{f}}, 205^{\mathrm{a}}, 178.5^{\mathrm{h}}$ & $210^{\mathrm{e}}, 206-268^{\mathrm{g}}, 202.4^{\mathrm{i}}, 245^{\mathrm{j}}, 188-245^{\mathrm{k}}, 204-210^{\mathrm{l}}$ \\
\hline $\mathbf{G}$ & 94.66 & - & - \\
\hline $\mathbf{E}$ & 259.28 & - & - \\
\hline $\mathbf{B} / \mathbf{G}$ & 2.199 & - & - \\
\hline $\mathbf{V}$ & 0.386 & - & - \\
\hline $\boldsymbol{\zeta}$ & 0.5883 & - & - \\
\hline $\mathbf{A}$ & 0.8784 & - & - \\
\hline
\end{tabular}

In Table 2, we summarized the calculated values of elastic parameters at different pressures up to $50 \mathrm{GPa}$. Under applied pressure, the calculated elastic constants for $\mathrm{w}-\mathrm{GaN}$ are found to satisfy the mechanical stability conditions up to $32 \mathrm{GPa}$, and from $44.70-50 \mathrm{GPa}$. It was observed that the stiffness constants deviate from the stability criteria Eq. (1) when pressure increased from $33.40-44.60 \mathrm{GPa}$. This implies that $\mathrm{w}-\mathrm{GaN}$ material is mechanically unstable within this region. This might be as a result of phase transition from wurtzite structure to rock-salt phase. This trend is consistent with the reported results by Serrano et al. [25], showing by the Rama scattering characterization that the phase transition from wurtzite to rock-salt appeared within a pressure ranging from 42 to $49 \mathrm{GPa}$. Figure 1 shows the pressure dependence of the elastic constants. From these plots, it is clear that all calculated hexagonal stiffness constants have positive values and increase linearly in a pressure range of 0 - $32 \mathrm{GPa}$. 
Table 2. Calculated elastic constants $\mathrm{C}_{\mathrm{ij}}$ (in GPa), Young's modulus E (in GPa), shear modulus G (in GPa), bulk modulus B (in GPa), Pugh's ratio (v), Zener anisotropy factor (A) and Kleinman parameter $(\zeta$ ) for w-GaN under different pressures.

\begin{tabular}{|c|c|c|c|c|c|c|c|c|c|c|c|c|c|}
\hline $\mathbf{P}$ & $\begin{array}{l}C_{11} \\
(\mathbf{G P a})\end{array}$ & $\begin{array}{l}C_{12} \\
(\mathbf{G P a})\end{array}$ & $\begin{array}{l}C_{13} \\
(\mathbf{G P a})\end{array}$ & $\begin{array}{l}C_{33} \\
(\mathbf{G P a})\end{array}$ & $\begin{array}{l}C_{44} \\
(\mathbf{G P a})\end{array}$ & $\begin{array}{l}C_{66} \\
(\mathrm{GPa})\end{array}$ & $\begin{array}{l}\text { B } \\
(\text { GPa })\end{array}$ & $\begin{array}{l}\text { G } \\
\text { (GPa) }\end{array}$ & $\begin{array}{l}E \\
(\text { GPa })\end{array}$ & B/G & $\mathbf{V}$ & $\zeta$ & $\mathbf{A}$ \\
\hline 0.00 & 344.46 & 157.43 & 122.69 & 379.36 & 82.15 & 93.52 & 208.20 & 94.66 & 259.28 & 2.199 & 0.386 & 0.5883 & 0.878 \\
\hline 10.0 & 398.73 & 194.06 & 156.31 & 436.70 & 91.02 & 102.33 & 249.72 & 104.07 & 287.00 & 2.399 & 0.4029 & 0.6137 & 0.884 \\
\hline 20.0 & 448.64 & 228.75 & 188.75 & 488.92 & 98.87 & 109.95 & 288.74 & 112.21 & 311.10 & 2.573 & 0.4148 & 0.6333 & 0.899 \\
\hline 30.0 & 495.52 & 262.07 & 220.37 & 537.59 & 106.01 & 116.72 & 326.03 & 119.49 & 332.39 & 2.728 & 0.4238 & 0.6491 & 0.908 \\
\hline 32.0 & 507.83 & 271.81 & 221.93 & 559.83 & 107.38 & 118.01 & 334.08 & 122.17 & 339.36 & 2.734 & 0.437 & 0.6544 & 0.909 \\
\hline 33.4 & 19.94 & -208.92 & 603.64 & -1392.52 & 121.18 & 114.45 & 78.99 & 32.93 & 271.76 & 2.398 & 0.1873 & 5.9385 & 1.058 \\
\hline 33.5 & 20.99 & -208.06 & 603.66 & -1389.83 & 121.29 & 114.52 & 79.70 & 33.16 & 272.83 & 2.403 & 0.1912 & 6.1053 & 1.059 \\
\hline 33.6 & 22.01 & -207.18 & 603.60 & -1387.11 & 121.44 & 114.59 & 80.39 & 33.41 & 273.85 & 2.406 & 0.1949 & 6.283 & 1.060 \\
\hline 33.7 & 23.17 & -206.21 & 603.56 & -1383.94 & 121.63 & 114.69 & 81.19 & 33.71 & 275.04 & 2.408 & 0.1991 & 6.500 & 1.061 \\
\hline 33.8 & 24.11 & -205.40 & 603.67 & -1381 & 121.70 & 114.75 & 81.88 & 33.89 & 276.05 & 2.416 & 0.2028 & 6.6896 & 1.062 \\
\hline 33.9 & 25.17 & -204.49 & 603.64 & -1378.68 & 121.84 & 114.83 & 82.61 & 34.14 & 227.10 & 2.419 & 0.2066 & 6.9193 & 1.063 \\
\hline 36.5 & -79.67 & -287.89 & 702.44 & -1417.26 & 123.05 & 104.11 & 75.26 & 18.60 & 254.96 & 4.046 & 0.2244 & 2.1025 & 1.201 \\
\hline 36.6 & -88.88 & -294.64 & 707.49 & -1420.24 & 125.18 & 102.88 & 73.40 & 17.22 & 250.88 & 4.262 & 0.2193 & 2.019 & 1.216 \\
\hline 36.7 & 1137.51 & 96.59 & -886.13 & -174.46 & 136.79 & 88.46 & -156.39 & 200.72 & 348.29 & -0.779 & 0.9686 & 0.234 & 1.546 \\
\hline 36.8 & 1130.93 & 955.69 & -882.52 & -175.38 & 136.95 & 87.62 & -156.65 & 199.76 & 344.96 & -0.784 & 0.9685 & 0.893 & 1.562 \\
\hline 40.0 & 905.79 & -851.11 & 104.18 & 141.98 & 141.98 & 47.08 & -144.61 & 167.13 & 187.20 & -0.865 & 0.`9882 & 0.9358 & 3.020 \\
\hline 41.0 & 1032.52 & 992.08 & -927.75 & 5.88 & 143.12 & 20.22 & -1299.60 & 150.63 & 80.91 & -0.860 & 1.000 & 0.9736 & 7.078 \\
\hline 43.0 & 1059.17 & 1122.75 & $\begin{array}{l}- \\
1048.08\end{array}$ & 212.20 & 145.53 & -31.79 & -106.20 & 85.37 & -126.17 & -1.243 & 0.9846 & 1.0394 & 4.577 \\
\hline 44.0 & -288.03 & -231.80 & 641.56 & -1079.45 & 157.58 & -28.12 & 49.82 & -103.48 & -146.36 & -0.481 & 1.602 & 0.8639 & -5.603 \\
\hline 44.5 & -316.91 & -234.52 & $654 . .38$ & -1069.70 & 158.37 & -41.19 & 49.82 & -135.00 & -246.15 & -0.369 & 1.987 & 0.8159 & -3.844 \\
\hline 44.6 & -324.30 & -236.69 & 659.05 & -1073.36 & 158.47 & -43.31 & 49.43 & -139.37 & -270.76 & -0.354 & 2.0903 & 0.8084 & -3.658 \\
\hline 44.7 & 1345.10 & 1173.96 & 373.96 & 701.58 & 125.88 & 85.57 & 708.52 & 141.54 & 316.72 & 5.000 & 0.8506 & 0.9127 & 1.471 \\
\hline 50.0 & 1437.44 & 1231.30 & 388.13 & 719.11 & 128.40 & 103.07 & 739.40 & 154.45 & 377.67 & 4.787 & 0.8321 & 0.9012 & 1.2457 \\
\hline
\end{tabular}


The pressure dependence of bulk modulus $(B)$, shear modulus $(G)$ and Young's modulus $(E)$ is presented in Figure 2. The Young's modulus, (which is the resistance to uniaxial tension) is used to indicate the stiffness of solids. This means that higher values of $E$ represent higher degree of stiffness of a material [26-28]. From our results, we noticed that pressure has significant effect on $E$. Thus, the Young's modulus of w-GaN shows a monotonic increase from $259.28-339.60 \mathrm{GPa}$ as pressure increased from $0-32 \mathrm{GPa}$. Beyond this pressure, the Young's modulus was observed to be fluctuating with increase in pressure as shown in Table 2. This might be as a result of phase transition.

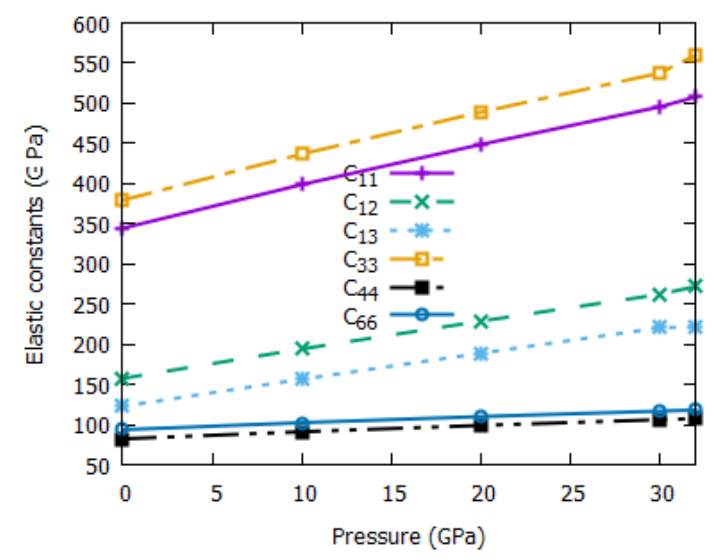

Figure 1. Variations of elastic constants $\left(\mathrm{C}_{\mathrm{ij}}\right)$ of $\mathrm{w}-\mathrm{GaN}$ with pressure.

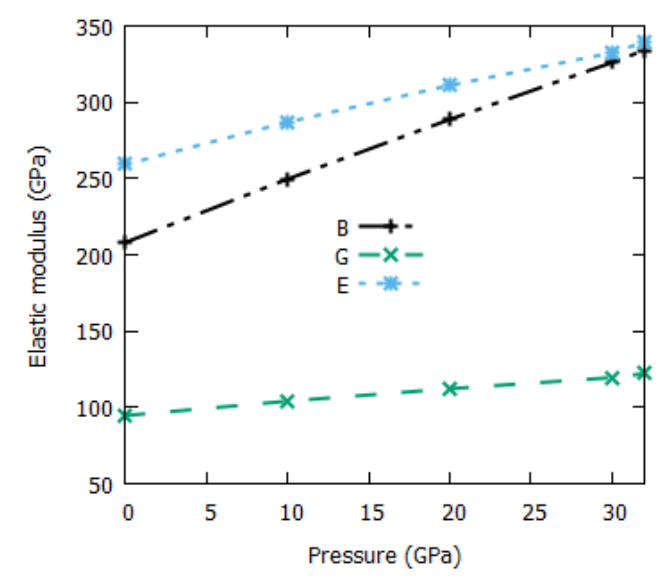

Figure 2. Variations of elastic modulus of w-GaN with pressure.

Bulk modulus determines a material's resistance to external deformation. It provides information on the physics of material bonding [29, 30]. It is evaluated as the average of Voigt modulus $B_{\mathrm{v}}$ [31] and Reuss modulus $B_{\mathrm{R}}$ [32]. For hexagonal crystals, $B_{\mathrm{v}}$ and $B_{\mathrm{R}}$ are related to the elastic constants $C_{\mathrm{ij}}$ as [33]

$$
\begin{aligned}
& B_{v}=\frac{1}{9}\left(4 C_{13}+2\left(C_{11}+C_{12}\right)+C_{33}\right) \\
& B_{R}=\frac{2 C_{13}^{2}-C_{33}\left(C_{11}+C_{12}\right)}{4 C_{13}-\left(2 C_{33} C_{11}+C_{12}\right)} \\
& B=\frac{1}{2}\left(B_{v}+B_{R}\right)
\end{aligned}
$$


The value of $B$ obtained at zero pressure is consistent with both experiment and theoretical results $[5,18]$ as captured in Table 1 . Under applied pressure, results obtained show that $B$ increases with increasing pressure up to 32 GPA as represented in Figure 2. From Table 2, it is clear that as the pressure is increased to $33.40 \mathrm{GPa}$, the bulk modulus decreased from 334.08 to $78.99 \mathrm{GPa}$ and continued to increase and decrease and finally increased as pressure is increased to $50 \mathrm{GPa}$. It was observed that w$\mathrm{GaN}$ is mechanically unstable in this region whereas the bulk modulus fluctuates.

In addition, shear modulus $G$ provides information on the resistance to change in shape initiated by a shearing force. $G$ is evaluated as the average of the Voigt modulus $G_{v}$ and the Reuss modulus $G_{R}$, from the relation: $G=\frac{1}{2}\left(G_{v}+G_{R}\right)$. For hexagonal crystals, $G_{v}$ and $G_{R}$ are connected to the elastic constants $C_{\mathrm{ij}}$ by the relations [33]:

$$
\begin{aligned}
& G_{v}=\frac{1}{30}\left(C_{11}+C_{12}\right)+\frac{1}{15}\left(C_{33}-2 C_{13}\right)+\frac{2}{5}\left(C_{44}+C_{66}\right) \\
& G_{R}=\frac{5\left[\left(C_{11}+C_{12}\right) C_{33}-2 C_{13}\right]^{2} C_{44} C_{66}}{12 B_{v} C_{44} C_{66}+4\left[\left(C_{11}+C_{12}\right) C_{33}-2 C_{13}\right]^{2}\left(C_{44}+C_{66}\right)} \\
& B_{v}=\frac{1}{9}\left(4 C_{13}+2\left(C_{11}+C_{12}\right)+C_{33}\right)
\end{aligned}
$$

It is well known that Vicker's hardness $\left(H_{v}=2\left(\left(\frac{G}{B}\right)^{2} G\right)^{0.585}-3\right)$ is related to the shear and bulk modulus [34] of a material. Therefore, the modulus can be used to predict the hardness of a material. From this relation, it means that high value of Pugh's ratio $(B / G)$ denotes low values of $H_{v}$, and vice versa. One can convincingly say that: $B, G$ and $H_{v}$, for any material have important effect on its technological applications [9]. Therefore, increase in $G$ increases the hardness of a solid material as long as $B$ remains constant, and vice versa. It was observed that the shear modulus $G$ increased montonically from $94.66-122.17 \mathrm{GPa}$ as the pressure increases from $0-32 \mathrm{GPa}$ as displayed in Figure 2. This portrays that the hardness $H_{v}$ of w-GaN tends to increase as pressure is increased from $0-32 \mathrm{GPa}$. In Table 2, it can be noticed that as pressure increased to $33.4 \mathrm{GPa}, G$ decreased to $32.93 \mathrm{GPa}$ and began to increase again, and then decreased to negative values before finally increased to $154.45 \mathrm{GPa}$ as the pressure goes up to $50 \mathrm{GPa}$. This suggests that due to phase transition that might have occurred from 33.4 - 44.6 GPa, w-GaN becomes unstable and posses negative shear modulus.

The mechanical behavior such as brittleness and ductility of a material are very significant for their technological applications. To determine these two extreme mechanical behaviors, the Pugh's ratio $B / G$ [35] is used as an indicator. If $B / G$ is greater than the threshold value 1.75 , the material is seen to exhibit a ductile behavior, while values less than this threshold indicate materials with brittle behavior. At zero pressure, the Pugh's ratio of $\mathrm{w}-\mathrm{GaN}$ is equal to 2.199 , above the threshold value of 1.75 . This result means that this material exhibits ductile behavior at zero pressure. Results obtained at high pressure for $B / G$ are plotted against pressure in Figure 3. As displayed in this figure, the Pugh's ratio increased linearly as pressure increases from $0 \mathrm{GPa}$ to $32 \mathrm{GPa}$. In Table 2, it can be observed that beyond the pressure of $32 \mathrm{GPa}$, the Pugh's ratio decreased and increased with increasing pressure, then decreased to negative values as pressure increases from $36.70-44.60 \mathrm{GPa}$, and finally increased with increasing pressure up to $50 \mathrm{GPa}$. These results suggest that in the region where Pugh's ratio is negative, w-GaN is prone to brittle behavior.

Theoretically, Poisson ratio is the ratio of the transverse strain to longitudinal strain. It is utilized to reflect the stability of materials against shear and gives information about the type of bonding forces $[36,37]$. The larger the Poisson's ratio is, the better is the plasticity of a material. For ionic materials v 
$=0.25$, covalent materials $\mathrm{v}=0.1$, and values from 0.25 to 0.5 signify that a central force exists in the solid material. In this study, the Poisson's ratio begins with 0.386 at zero pressure and increased to 0.437 as pressure increased to $32 \mathrm{GPa}$, as displayed in Figure 3. This signifies that central forces are predominant in $\mathrm{w}-\mathrm{GaN}$ material. This result is consistent with previous studies.

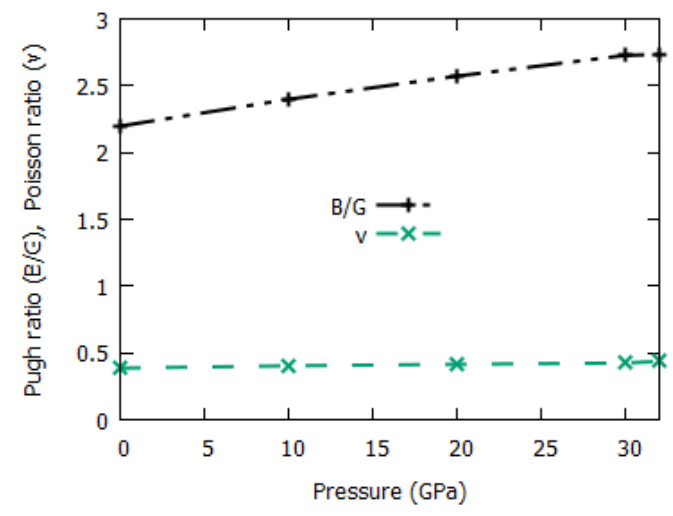

Figure 3. Variations of Pugh's ratio and Poisson's ratio of w-GaN with pressure.

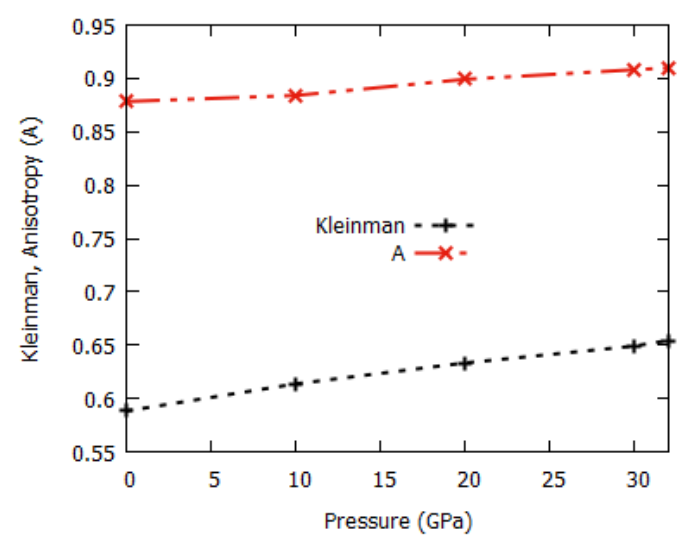

Figure 4. Variations of Kleinman parameter and Zener anisotropy factor of w-GaN with pressure.

Additionally, Zener anisotropy factor $(A)$ reveals information about degree of anisotropy in solid materials [38]. It has potential to influence micro-cracks in minerals; as such it becomes vital in material engineering. $A=1$ implies the material under study is elastically isotropic, otherwise the material is anisotropic. The elastic anisotropy can be evaluated by the relation: $A=2 C_{44} /\left(C_{11}-C_{12}\right)$. The value obtained for $A$ at zero pressure is 0.878 , indicating that $\mathrm{w}-\mathrm{GaN}$ exhibits an anisotropic semiconductor. The pressure dependence of $A$ is displayed in Figure 4. When the pressure is increased from zero to 32 $\mathrm{GPa}$, it was observed that $A$ increased continuously from 0.878 to 0.909 . This implies that w-GaN is an anisotropic material under high-pressure.

Kleinman parameter $(\zeta)$ portrays the relative positions of cation and anion lattices under volume conserving strain deformations. It is calculated by the equation [39]: $\zeta=\left(C_{11}+8 C_{12}\right) /\left(7 C_{11}+2 C_{12}\right)$. Reducing bond stretching in a material leads to $\zeta=1$, while reducing bond bending leads to $\zeta=0$. At zero pressure, the Kleinman parameter for $\mathrm{w}-\mathrm{GaN}$ is equal to 0.5883 as displayed in Table 2. Results obtained for $\zeta$ are plotted against pressure in Figure 4. It is clear that as pressure is increased up to 32 
$\mathrm{GPa}, \zeta$ grows monotonically. This suggests that, there is a decrease in bond bending as pressure is increased from $0 \mathrm{GPa}$ to $32 \mathrm{GPa}$. However, around (33.4 - 44.6) GPa, some unrealistic values of $\zeta$ were obtained. This might be a result of phase transition around these pressures.

\section{Conclusions}

In this work, we described the elastic and mechanical properties of $\mathrm{w}-\mathrm{GaN}$ under high pressure up to $50 \mathrm{GPa}$. The results obtained on these properties at zero pressure are consistent with both the experimentally and theoretically calculated values. It was observed that the structure of $\mathrm{GaN}$ was stable under pressure up to $32 \mathrm{GPa}$, and all calculated elastic parameters increased linearly with the increase of pressure. It was also discovered that by applying pressure one can improve the ductility of the structure of GaN.

\section{Acknowledgements}

The authors acknowledge the Federal University of Petroleum Resources Effurun for providing the Mini Workstation used for the calculations and also QuantumATK for access to the VNL-ATK software package.

\section{References}

[1] S. Nakamura, M. Senoh, T. Mukai, High-power InGaN/GaN Double-heterostructure Violet Light Emitting Diodes, Appl. Phys. Lett, Vol. 62, 1993, pp. 2390-2392, https://doi.org/10.1063/1.109374.

[2] A. Sadao, Properties of Group-IV, III-V and II-VI Semiconductors, Hoboken (USA) Wiley\&Sons, 2005.

[3] S. Nakamura, In Proceedings of International Symposium, Blue Laser and Light Emitting Diodes, 1996.

[4] F.S. Saoud, J.C. Plenet, L. Louail, D. Maouche, Mechanism of the Phase Transition in GaN Under Pressure up to 100 GPa, Comp. Theo. Chem, Vol. 964, 2011, pp. 65-71, https://doi.org/10.1016/j.comptc.2010.11.037.

[5] M. Yamaguchi, T. Yagi, T. Azuhata, T. Sota, K. Suzuki, S. Chichibu, S. Nakamura, Brillouin Scattering Study of Gallium Nitride: Elastic Stiffness Constants, J. Phys.: Condens. Matter, Vol. 9, 1997, pp. 241, https://doi.org/10.1088/0953-8984/9/1/025.

[6] S. Nakamura, T. Mukai, M. Senoh, Candela-class High-brightness InGaN/AlGaN Double-heterostructure BlueLight-Emitting Diodes, Appl. Phys. Lett, Vol. 64, 1994, pp. 1687-1689, https://doi.org/10.1063/1.111832.

[7] S.Y. Davydov, Evaluation of Physical Parameters for the Group III Nitrates: BN, AlN, GaN, and InN, Semiconductors, Vol. 36, 2002, pp. 41-44, https://doi.org/10.1134/1.1434511.

[8] A.F. Wright, Elastic Properties of Zinc-blende and Wurtzite AlN, GaN, and InN, J. Appl. Phys, Vol. 82, 1997, pp. 2833-2839, https://doi.org/10.1063/1.366114.

[9] N. Lebga, S. Daoud, X.-W. Sun, N. Bioud, A. Latreche, Mechanical and Thermophysical Properties of Cubic RockSalt AlN Under High Pressure, J. Electronic Mat, Vol. 47, 2018, pp. 3430-3439, https://doi.org/10.1007/s11664018-6169-x.

[10] A. Polian, M. Grimsditch, I. Grzegory, Elastic Constants of Gallium Nitride, J. Appl. Phys, Vol. 79, 1996, pp. 3343-3344, https://doi.org/10.1063/1.361236.

[11] T. Li-Na, H. Cui-E, Y. Bai-Ru, C. Xiang-Rong, First-principles Calculations of Structure and High Pressure Phase Transition in Gallium Nitride, Chin. Phys, Vol. 16, 2007, pp. 3772-3776, https://doi.org/10.1088/10091963/16/12/036.

[12] M.E. Sherwin, T.J. Drummond, Predicted Elastic Constants and Critical Layer Thicknesses for Cubic Phase AlN, GaN, and InN on $\beta$-SiC, J. Appl. Phys, Vol. 69, 1991, pp. 8423-8425, https://doi.org/10.1063/1.347412. 
[13] J. Nord, K. Albe, P. Erhart, K. Nordlund, Modelling of Compound Semiconductors: Analytical Bond-order Potential for Gallium, Nitrogen, Gallium Nitride, J. Phys.: Condens. Matter, Vol. 15, 2003, pp. 5649, https://doi.org/10.1088/0953-8984/15/32/324.

[14] Atomistix ToolKit 2017.2. Quantumwise A/S, www.quantumwise.com.

[15] V.A. Savastenko, A.U. Sheleg, Study of the Elastic Properties of Gallium Nitride, Physica Status Solidi (a), Vol. 48, 1978, pp. K135-K139, https://doi.org/10.1002/pssa.2210480253.

[16] M. Born, On the Stability of Crystal Lattices, Math. Proceedings of the Cambridge Philosophical Society, Vol. 36, 1940, pp. 160-172, https://doi.org/10.1017/S0305004100017138.

[17] F. Mouhat, F.-X. Coudert, Necessary and Sufficient Elastic Stability Conditions in Various Crystal Systems, Phys. Rev. B, Vol. 90, 2014, pp.22410, https://doi.org/10.1103/physrevb.90.224104.

[18] M.-M. Soumelidou, I. Belabbas, J. Kioseoglou, P. Komninou, J. Chen, T. Karakostas, Strain and Elastic Constants of GaN and InN, Comp. Condens. Matter, Vol. 10, 2017, pp. 25-30, https://doi.org/10.1016/j.cocom.2017.02.001.

[19] K. Kim, W.R.L. Lambrecht, B. Segall, Elastic Constants and Related Properties of Tetrahedrally Bonded BN, AlN, GaN, and InN, Phys. Rev. B, Vol. 53, 1996, pp. 16310-16326, https://doi.org/10.1103/physrevb.53.16310.

[20] M. Ueno, M. Yoshida, A. Onodera, O. Shimomura, K. Takemura, Stability of the Wurtzite-type Structure Under High Pressure: GaN and InN, Phys. Rev. B, Vol. 49, 1994, pp. 14-21, https://doi.org/10.1103/physrevb.49.14.

[21] T. Tsuchiya, K. Kawamura, O. Ohtaka, H. Fukui, T. Kikegawa, Precise Measurement of Equation-of-State and Elastic Properties for $\mathrm{GaN}$ up to $16 \mathrm{GPa}$, Solid State Comm, Vol. 121, 2002, pp. 555-559, https://doi.org/10.1016/S0038-1098(01)00492-6.

[22] P. Perlin, C. Jauberthie-Carillon, J.P. Itie, A. San Miguel, I. Grzegory, A. Polian, Raman Scattering and X-rayAbsorption Spectroscopy in Gallium Nitride Under High Pressure, Phys. Rev. B, Vol. 45, 1992, pp. 83-89, https://doi.org/10.1103/physrevb.45.83.

[23] P. Perlin, C. Jauberthie-Carilln, J.P. Itie, A. San Higuel, I. Grzecory, A. Wlian, High Pressure Phase Transition in Gallium Nitride, High Press. Res, Vol. 7, 1991, pp. 96-98. https://doi.org/10.1080/08957959108245516.

[24] M. Leszczynski, T. Suski, P. Perlin, H. Teisseyre, I. Grzegory, M. Bockowski, J. Major, Lattice Constants, Thermal Expansion and Compressibility of Gallium Nitride, J. Phys. D: Appl. Phys, Vol. 28, No. 4A, 1995, pp. A149A153, https://doi.org/10.1088/0022-3727/28/4a/029.

[25] J. Serrano, A. Rubio, E. Hernández, A. Muñoz, A. Mujica, Theoretical Study of the Relative Stability of Structural Phases in Group-III Nitrides at High Pressures, Phys. Rev. B, Vol. 62, 2000, pp. 1661216623, https://doi.org/10.1103/physrevb.62.16612.

[26] S. Wang, J.-X. Li, Y.-L Du, C. Cui, First-Principles Study on Structural, Electronic and Elastic Properties of Graphene-like Hexagonal Ti2C Monolayer, Comp. Mat. Sc, Vol. 83, 2014, pp. 290293, https://doi.org/10.1016/j.commatsci.2013.11.025.

[27] L. Feng, N. Li, M. Yang, Z. Liu, Effect of Pressure on Elastic, Mechanical and Electronic Properties of WSe2: A FIrst-Principles Study, Mat. Res. Bull, Vol. 50, 2014, pp. 503-508, https://doi.org/10.1016/j.materresbull.2013.11.016.

[28] L. Bing, L. Rong-Feng, Y. Yong, Y. Xiang-Dong, Characterisation of The High-Pressure Structural Transition and Elastic Properties in Boron Arsenic, Chin. Phys. B, Vol. 19, 2010, pp. 076201, https://doi.org/10.1088/16741056/19/7/076201.

[29] S. Bensalem, M. Chegaar, D. Maouche, A. Bouhemadou, Theoretical Study of Structural, Elastic and Thermodynamic Properties of CZTX (X=S and Se) Alloys, J. Alloys and Compounds, Vol. 589, 2014, pp. 137142, https://doi.org/10.1016/j.jallcom.2013.11.113.

[30] M. Guemou, A. Abdiche, R. Riane, R. Khenata, Ab Initio Study of the Structural, Electronic and Optical Properties of Bas and BN Compounds and $\mathrm{BN}_{\mathrm{x}} \mathrm{As}_{1-\mathrm{x}}$ alloys, Phys. B: Condens. Matter, Vol. 436, 2014, pp. 3340, https://doi.org/10.1016/j.physb.2013.11.030.

[31] W. Voigt, Textbook of crystal physics, 962, Leipzig: Teubner, 1928.

[32] A. Reuss, Calculation of the Yield Point of Mixed Crystals Based on the Plasticity Condition for Single Crystals, ZAMM-J. Appl. Math. Mech, Vol. 9, 1992, pp. 49-58.

[33] E. Schreiber, O.L. Anderson, N. Soga, Elastic Constants and their Measurement, New York: McGraw-Hill, 1973, pp. 1-79. 
[34] X.-Q. Chen, H. Niu, D. Li, Y. Li, Modeling Hardness of Polycrystalline Materials and Bulk Metallic Glasses, Intermetallics, Vol. 19, 2011, pp. 1275-1281, https://doi.org/10.1016/j.intermet.2011.03.026.

[35] S.F. Pugh, Relation between the Elastic Moduli and the Plastic Properties of Polycrystalline Pure Metals, Phil. Mag. J. Sc, Vol. 45, 1954, pp. 823-843, https://doi.org/10.1080/14786440808520496.

[36] G.N. Greaves, A.L. Greer, R.S. Lakes, T. Rouxel, Poisson's Ratio and Modern Materials, Nature Materials, Vol. 10, 2011, pp. 823-837, https://doi.org/10.1038/nmat3134.

[37] Y. Cao, J. Zhu, Y. Liu, Z. Nong, Z. Lai, First-Principles Studies of the Structural, Elastic, Electronic and Thermal properties of $\mathrm{Ni}_{3} \mathrm{Si}$, Comp. Mat. Sc, Vol. 69, 2013, pp. 40-45, https://doi.org/10.1016/j.commatsci.2012.11.037.

[38] C. Zener, Theory of Strain Interaction of Solute Atoms, Phys. Rev, Vol. 74, 1948, pp. 639647, https://doi.org/10.1103/physrev.74.639.

[39] A.W. Harrison, Electronic Structure and Properties of Solids, New York: Dover, 1989. 